\title{
LESS IS MORE, MINIMALISME ET MUSIQUE
}

\author{
Lionel Detry ${ }^{1}$
}

L'émergence de la musique minimaliste au début des années soixante peut s'envisager dans un mouvement plus global d'éloge du moins rassemblé derrière l'expression: less is more. Ce sujet dépasse les limites du champ artistique comme on peut le voir dans le volume des Cahiers de Médiologie (Dagonet, 2000) qui y est consacré. Je propose ici une analyse de la musique minimaliste à partir du cadre médiologique proposé par Oudin (2000) dans son article consacré à l'architecture minimale. Ce courant musical fait partie de ce mouvement " moins ", notamment par le matériau utilisé pour la composition. J'identifie plusieurs caractéristiques qui permettent de mieux comprendre et de définir cette musique au regard de ce mouvement plus global dans la société du XXe siècle. J'en propose également un éclairage à la lumière du concept de postmodernité.

1 Lionel Detry est Assistant et Doctorant à l'École de communication et membre du Groupe de Recherche en Médiation des Savoirs (GReMS) à l'Université catholique de Louvain.

Recherches en communication, $\mathrm{n}^{\circ} 38$ (2012). 


\section{L'architecture et le vide}

Dans son article sur les architectures minimales, Bernard Oudin (2000) présente l'éloge du moins célébré par l'architecte Mies van der Rohe et ses contemporains : less is more. L'auteur rend compte d'une constante dans la construction d'édifices (et, peut-être, dans l'art en général) : «l'approche vers une phase de surcharge est progressive alors que la phase de dépouillement survient brutalement » (ibid., p. 63). Tout se passe comme si un style d'architecture ajoutait un élément sur un autre en les additionnant telle une maison qui se charge des objets amassés durant toute une vie. Un exemple en est le style classique qui a évolué petit à petit jusqu'au rococo. À l'opposé, l'épuration, le passage au moins se fait de manière plus franche et relève généralement d'un mouvement radical qui décide de faire table rase sur le passé. Oudin met cependant en garde contre un présupposé hâtif qui voudrait considérer le plus et le moins au regard respectivement du conservatisme et de la modernité. Certains styles surchargés ont été jugés décadents alors que d'autres du côté du moins (l'Art Nouveau, par exemple) d'abord estimés comme des avant-gardes ont ensuite été jugés décadents à leur tour. Il s'agit donc plutôt d'un changement de mode et de mentalité.

Il n'est donc pas question d'opposer les deux approches sur la question de l'innovation. Cependant, leur démarche n'est pas similaire. Le plus est ancré dans des références culturelles historiques et s'attache à un culte du beau hors des réalités quotidiennes. Le moins au contraire s'est basé sur des techniques neuves et s'est inspiré des évolutions $\mathrm{du}$ quotidien telles que les nouvelles machines pour rechercher une nouvelle définition de la beauté en art.

L'auteur défend ensuite la méprise de certaines oppositions trop faciles entre le plus et le moins (irrationnel vs rationnel ; folie vs rigueur...) avant de replonger dans l'histoire pour y trouver les origines de ce culte du moins. Il imagine d'abord sa naissance à l'Antiquité en pensant aux temples grecs, par exemple. Toutefois, c'est la découverte de ces vestiges tels qu'ils sont apparus qui fait croire cela. En effet, ces temples étaient, à l'époque, colorés de manière extrême et ornementés de divers objets et offrandes. Néanmoins, la découverte du style dorique au XVIII' siècle a marqué en profondeur l'architecture de l'époque. Ce style typiquement épuré a inspiré de nombreux constructeurs et, notamment, les Américains qui l'ont édifié en style officiel. 
Mais cet engouement minimaliste n'est rien par rapport à l'architecture du $\mathrm{XX}^{\mathrm{e}}$ siècle, "siècle par excellence du minimalisme " (ibid., p. 66). Elle introduit réellement une rupture avec le passé par un changement radical qui correspond à l'importance des transformations dans la société de l'époque. Sa seule référence avouée est l'industrie. « L'architecture moderne s'est voulue industrielle dans ses techniques, son esprit et ses formes » (ibid., p. 67). Cette volonté aurait pu se traduire par une utilisation des nouvelles techniques pour reproduire des styles passés ou pour reproduire le style en série, mais ce n'est pas ce qui s'est produit lors de cet engouement minimaliste.

Les critiques se lèvent pour déclarer cette architecture comme fonctionnelle. Or, le minimalisme est plus souvent hissé au rang d'une véritable esthétique. C'est un choix peu évident pour l'auteur qui donne l'exemple des couvents cisterciens (ibid., p. 68). Les moines qui ont bâti ces édifices n'ont jamais voulu faire de l'art. Au contraire, les choix de construction ont plutôt porté sur la volonté d'évacuer le beau des bâtiments religieux. Leur spiritualité a concrétisé dans la pierre les obligations de renoncement et d'austérité. Néanmoins, sans le savoir, ils ont créé des lieux qui sont aujourd'hui visités pour leurs attraits architecturaux et qui ont inspiré les architectes du $\mathrm{XX}^{\mathrm{e}}$ siècle. Ceux-ci partagent, en effet, un goût certain pour la pureté et pour le rejet de l'ornementation. Mais Oudin trouve cependant que la vie proposée par ces nouveaux bâtisseurs n'est pas des plus riantes et, peut-être, évacuaient-ils aussi l'art de leurs préoccupations.

C'est l'avis d'Adolf Loos, le théoricien du moins en architecture. Très critique envers ses contemporains, selon lui : "Seule une très petite partie de l'architecture appartient à l'art : la tombe et le monument » (ibid., p. 69). L'auteur de l'article est moins catégorique et se rend compte que le minimalisme a quand même produit quelques chefs-d'œuvre que l'on doit au talent de certains artistes. « Le drame du minimalisme, c'est son succès » (ibid., p. 70). L'intérêt du minimalisme en architecture est justement qu'il soit moins présent. Tout comme une œuvre d'art acquiert de la valeur par son unicité et son originalité, « le moins (comme le plus) ne peut que gagner à demeurer une exception, tant il est vrai qu'en matière d'art, la première qualité d'une œuvre est sa rareté » (idem). 


\section{Musique et minimalisme}

On attribue souvent la naissance de la musique minimaliste (ou répétitive - ce qui n'est pas un meilleur terme pour définir ce courant) à Terry Riley. En 1964, In C jette les bases d'un courant qui sera porté par son auteur et trois autres compositeurs : La Monte Young, Steve Reich et Philip Glass. La base de cette musique est généralement un court motif mélodique qui se répète et évolue graduellement au fil de l'œuvre. Pour La Monte Young, c'est même souvent un seul son unique qui se développe tout au long de la pièce (un drone). Utilisant cette technique dès 1958 (Trio for Strings), on lui attribue aussi parfois la paternité de la musique minimaliste (Mussat, 2002, p. 142).

La musique répétitive veut se démarquer des avant-gardes. Elle s'inscrit en réaction face à l'indétermination de John Cage et veut réintroduire la communication avec le public qui se désintéressait de l'approche conceptuelle des compositeurs des années 50-60 (ibid., p. 141). Cette musique, influencée par le jazz, le rock et les musiques non-occidentales rencontrera son public dans des milieux qui se rapprochaient de ses idées (galeries d'art, universités...). Paradoxalement, c'est le succès auprès d'un plus large public qui empêchera pendant longtemps la musique minimaliste d'avoir une reconnaissance de la part de ses pairs (Potter, 2007).

\section{Système et répétition}

Ce bref aperçu de la musique minimaliste fait déjà entrevoir des liens évidents avec la question du less is more. La base de cette musique est toujours un matériau minime. Très souvent il se limite à un petit nombre d'éléments premiers. Les premières pièces répétitives de Steve Reich sont établies à partir d'un seul court fragment vocal (It's Gonna Rain en 1965 et Come out en 1966) et il développera cette même idée dans ses autres pièces jusqu'à Drumming (1971) qui commence avec un seul coup de percussion.

Ce qui fait la particularité de ces compositions, c'est la manière de combiner les éléments, d'agencer et d'assembler les notes qui permet un développement étendu. Chez Steve Reich, c'est le gradual process (Reich, 1974, pp. 9-11). Deux ou plusieurs patterns sont alignés à l'unisson et se décalent progressivement au cours de l'œuvre. Cela 
donne des rencontres harmoniques et rythmiques qui seront le cœur de la pièce de musique. Ce système était au départ voué à être joué par une machine car le compositeur n'imaginait pas pouvoir faire jouer ce déphasage par des humains. Néanmoins, il est déçu par la machine créée pour exécuter sa musique (The Phase Shifting Pulse Gate (ibid., p. 25)) en raison de son absolue perfection. Il se rend compte que, ce qui va être intéressant dans ce déphasage, c'est le jeu des musiciens. Les micro-variations insérées par l'interprétation rendront le résultat final plus musical.

Comme en architecture minimaliste, ce mouvement musical entretient un lien étroit avec l'innovation technique. Elle fait partie intégrante du projet parce qu'elle est une part de notre quotidien. Néanmoins, comme dans cet exemple de Reich, l'innovation n'est pas visée pour elle-même mais pour servir le projet artistique.

La manière d'agencer les séquences n'est pas entièrement systématique et Steve Reich assume la part d'intuition (ibid., p. vii) qui est nécessaire au processus compositionnel. Il y a un certain tri dans les processus possibles afin de déterminer ce qui rendra la pièce plus intéressante. Lorsque celui-ci est défini, l'œuvre se déroule et fait apparaître des rencontres surprenantes. Cette cassure du systématisme empêche le minimalisme de virer vers un penchant réductionniste.

Une constante dans la musique de Steve Reich est la longueur des œuvres. Pourquoi, dès lors, appeler " minimaliste » une musique qui dure si longtemps et qui contient tant de notes ? La durée de l'œuvre est justement nécessaire pour souligner la restriction des éléments de base (Gann, 2004, p. 299). Le temps permet de développer les possibilités de rencontres rythmiques, harmoniques... C'est cette durée qui permet aussi à l'auditeur de « rentrer » dans la musique, d'en comprendre la construction et d'expérimenter une autre attitude face au son. Cette répétitivité sur la longueur est souvent ce qui fascine le public ou qui énerve les critiques. Elle n'est pourtant pas à considérer comme une reproduction, une répétition d'éléments identiques, mais bien comme une répétition de l'identique autrement (Fink, 2005, p. 6). 


\section{Plateau et affects}

Les résultats des processus graduels de Steve Reich exercent une certaine fascination chez l'auditeur (et chez le compositeur également) tout comme les drones de La Monte Young. En se détournant d'une logique " classique » de tension, relâchement, climax, dénouement, le compositeur montre un intérêt pour ce que Deleuze et Guattari nomment le «plateau » (un terme emprunté à Bateson) : « une région continue d'intensités, vibrant sur elle-même, et qui se développe en évitant toute orientation sur un point culminant ou vers une fin extérieure » (Deleuze \& Guattari, 1998, p. 32 cité dans Cox \& Warner, 2004, p. 288). Cette production sonore permet à l'auditeur d'accéder à un état de transe ou de faire partir l'esprit loin. La volonté de provoquer des effets physiologiques est d'ailleurs explicite chez les compositeurs, notamment chez Glass (Mertens, 2004, p. 310-311). On peut retrouver aujourd'hui des traces de cet esprit dans la musique techno ou la musique de méditation qui doit avoir comme effet d'emmener l'auditeur dans un autre état de conscience et de présence (McClary, 2004, p. 295).

Les premiers minimalistes avaient sans doute perçu la puissance que ce type de musique pouvait avoir sur l'auditeur. La sociologie s'est attachée à montrer que les individus utilisaient la musique comme ressource pour se changer soi (DeNora, 2000). La neurologie s'est intéressée à ce pouvoir de la musique en montrant comment et pourquoi certaines personnes développaient des réactions très fortes à certaines musiques. En utilisant cette force, Oliver Sacks $(2007,2011)$ explique comment la musique est utilisée dans un but thérapeutique. Un exemple (cité dans DeNora, 2000, p. 151) illustre cette idée. Une femme atteinte de Parkinson fait le lien entre la perte de sa motricité « gracieuse » et la musique. Elle retrouve ses mouvements après avoir été « remusiquée».

\section{Silence et contemplation}

À la suite de la naissance de ce courant répétitif, un autre courant plus contemplatif s'est développé avec cette envie de minimalisme. Cette musique se rapproche plus de ce que nous avons vu au sujet de l'architecture minimaliste. Elle se présente en opposition par rapport aux avant-gardes et veut introduire une cassure franche avec cette période de la musique du $\mathrm{XX}^{\mathrm{e}}$ siècle. Elle désire " réintroduire le contenu émotionnel comme valeur fondamentale de la musique, dans 
le but avoué de renouer avec le public et de l'élargir en favorisant une communication directe» (Mussat, 2002, p. 146).

Un des grands architectes de ce mouvement est le compositeur estonien Arvo Pärt. À l'opposé de l'expérience de silence de John Cage $^{1}$, il réintroduit le continuum afin de faire ressentir une nouvelle expérience du temps. Ces œuvres simples, à première vue, ne cachent pas leur intention de provoquer des sentiments forts chez l'auditeur. Il n'est d'ailleurs pas anodin de signaler que cette musique minimaliste a été utilisée dans de nombreux films pour cette capacité d'évocation d'émotions. On peut penser à Michael Nyman dans The Piano (Campion, 1993) ou à György Ligeti dont la Musica Ricercata a été utilisée de manière dramatique dans Eyes Wide Shut de Stanley Kubrick (1999).

Comme pour l'architecture minimale, les critiques n'hésitent pas à définir cette musique comme fonctionnelle, comme une musique d'environnement. Brian Eno ne leur donnera pas tort en créant la musique ambient, une musique adaptée au lieu où elle est écoutée (et donc à son architecture). Dans le manifeste paru avec Music for Airports (1978), il écrit :

Une ambiance, c'est une atmosphère, une influence environnante, une coloration. Mon intention est de produire des morceaux originaux censés être réservés (mais pas exclusivement) à des moments et à des contextes particuliers, en vue de dresser un petit catalogue varié de musique environnementale qui puisse s'adapter à une vaste gamme d'humeurs et d'atmosphères. (Eno, 1996, p. 296 traduit dans Collectif, 1997, p. 9)

Mais si cette musique contemplative a bien une fonction, ce serait, comme les monastères, celle de se libérer l'esprit. Telle l'évocation du vide, cette musique tend à insuffler de la présence (d'où son caractère souvent religieux) par l'absence.

1 4'33"'est une œuvre entièrement silencieuse qui exemplifie la réflexion de Cage sur la place de la musique dans l'environnement quotidien (Cage, 1970). 


\section{Minimalisme et postmodernité}

L'ensemble de cette musique diffusée après 1965 a souvent été caractérisée de postmoderne (Mussat, 2002). Mais ce sont justement les spécificités de la musique minimale qui se retrouvent dans la musique qualifiée de postmoderne.

Dans son mémoire de licence en communication, Detry (2002), a développé une grille d'analyse de la postmodernité dans la musique. Appliquée à la musique des Beatles, cette grille a permis d'illustrer une expression musicale des différents concepts caractéristiques d'une société postmoderne (Lyotard, 1996 ; Ramaut-Chevassus, 1998). L'analyse effectuée met en évidence les liens existant entre musique et société. Partant des acquis de cette recherche, la musique minimale s'intègre parfaitement dans cette grille. Je m'appuie, une fois de plus, principalement sur la musique de Steve Reich pour son aspect typique du courant.

La musique postmoderne se caractérise par une triple ouverture : une ouverture de l'espace-temps-son, du système de communication et de la production du sens. L'ouverture de l'espace-temps-son est, premièrement, remarquable par différents aspects. La musique minimaliste élargit la panoplie des instruments utilisés au-delà de l'orchestre classique (percussions africaines, clappements de mains) et se tourne facilement vers les nouvelles technologies pour produire des sons (machine créée pour la composition, synthétiseurs) ou pour faire des collages sur bandes magnétiques (It's Gonna Rain). Elle ne renie pas le passé et n'hésite pas à faire usage de certaines formes classiques (symphonies et opéras de Philip Glass) et à une harmonie tonale. Il y a également souvent un aspect mystique ou religieux dans ces musiques (La Monte Young ou Arvo Pärt). Elle s'inspire fortement des musiques non-occidentales (percussions africaines et balinaises pour Reich, musique indienne pour Glass, entre autres). Wim Mertens (2004, p. 308) voit dans ce trait caractéristique non une fondation de ces musiques mais un symptôme de nos sociétés contemporaines d'annexer une autre culture en la transposant dans un nouveau cadre et, dès lors, la vider de sa substance. Il y a également un lien fort de la musique minimaliste avec les musiques actuelles de l'époque (Glass crée sa symphonie Heroes à partir d'un album de David Bowie). 
Ensuite, elle provoque une ouverture du système de communication. Souvent, la composition annule le « sujet énonciateur au profit de la musique et du jeu répétitif » (Stoianova, 1977, p. 73). Comme dit plus haut, l'émotion comme facteur de communication est de retour. Le recours aux mélodies et à la tonalité prend le pas sur la sérialité et la recherche conceptuelle propre aux années cinquante. La transparence compositionnelle du processus graduel chez Reich tend également à susciter la curiosité et la compréhension de l'auditeur et à renouer le dialogue avec le compositeur. «You want to hear music that moves you, and if you don't, then you're not very curious to find out how it was put together » (Reich, 1974, p. vii). Selon Gann (2004, p. 302-303), un trait distinctif de la musique minimaliste est justement que l'auditeur peut facilement entendre la structure qui est à la base de la composition. Glass va jusqu'à dire que l'oeuvre n'est plus représentationnelle, elle ne vise qu'elle-même (Glass cité par Mertens, 2004, p. 309). L'écoute change alors également étant donné que la répétition d'un thème n'a pas lieu. Par conséquent, le désir des compositeurs est de développer une écoute purement sonore, la perception d'un événement-son.

Enfin, la musique postmoderne crée une ouverture dans la production du sens. Le sens d'une œuvre n'est pas prédéfini et, même, cette musique est particulièrement riche en interprétations. Le fait d'être non-représentationnelle aide à cette non-définition interne de l'œuvre. Le sens se trouve autant du côté de son créateur que de son récepteur. «Les lectures déterminées par la question "Que 'dit' ce processus musical répétitif ?" peuvent, bien sûr, se multiplier indéfiniment » (Stoianova, 1977, p. 74).

\section{Résonance et quotidien}

Dans l'histoire de la musique dite classique, le courant minimaliste occupe une place bien à part. Ses compositeurs ont été formés de manière classique mais ils se sont vite affranchis du chemin tracé pour s'échapper dans des contrées autres. Survenu légèrement après l'éclosion du rock'n'roll, le mouvement minimaliste a plusieurs choses en commun avec cette musique. Elle développe une énergie rythmique particulière qui fait lien avec le corps en proposant une expérience sonore qui est au centre de la musique. En jouant sur le rythme et la pulsation, Steve Reich explore l'aspect le plus physique de la musique par sa vibration directe avec le corps humain. Cet aspect direct est un 
des éléments qui a permis de renouer la communication avec le public qui avait déserté les avant-gardes. Cette audience curieuse a retrouvé de quoi satisfaire son besoin de comprendre pour mieux entendre. Le courant plus contemplatif a fourni une nouvelle expérience du temps en accroissant la lenteur et le vide dans un monde obsédé par la vitesse. Rosa estime que l'hyper-musicalisation de la vie quotidienne (le baladeur, par exemple) stimule des expériences d' " autorésonance » mais témoigne, en même temps, d'une non-résonance avec son environnement (Rosa, 2012, p. 140-141). Cette musique qui prend son temps peut être un chemin pour sortir de ce malaise.

Aujourd'hui, la musique minimaliste vit toujours avec ses fondateurs et quelques disciples qui ont continué les travaux des pionniers. On parle parfois, pour certains compositeurs, de postminimalisme (Schwarz, 1990). Cependant, la situation a légèrement changé. Les minimalistes ont finalement trouvé une reconnaissance dans le milieu de la musique classique et il semble probable que le courant le plus prolifique se soit déplacé vers la musique électronique minimaliste (notamment, la techno et, plus récemment, le dubstep).

La stratégie du moins chez les musiciens minimalistes leur a permis d'élargir la communication avec le public. Celle-ci est peut-être imputable à une présence de la logique minimaliste dans différents pans de notre quotidien (Dagonet, 2000). En sciences, l'ADN, avec seulement quatre lettres, permet de générer tous les humains existants ou possibles. Avec le système binaire informatique, le réductionnisme touche à la perfection : deux simples signes différenciés $(0$ et 1$)$ permettent de diffuser à peu près tout par la numérisation des données (texte, audio, vidéo...). Le développement de la langue a également un penchant pour le moins. Le succès des proverbes, aphorismes, maximes et, bien sûr, des slogans (publicitaires, politiques et autres) exprime bien cette possibilité d'en dire beaucoup avec une quantité limitée de mots (Debray, 2000). Il en est de même pour nos technologies de messagerie courte. Le texto ou le tweet demande à son auteur de faire preuve d'une certaine dose de créativité en le contraignant, respectivement, à 160 ou 140 caractères. Comme pour d'autres formes langagières, la règle stricte augmente la création plutôt que de la réduire : less is more une fois de plus. 
Ce minimalisme qui nous environne nous fait aussi entendre, au quotidien, la musique de notre minimaliste national, Wim Mertens, utilisée à des fins publicitaires (et de sonnerie !) dans le domaine des télécommunications...

\section{Références}

Cage, J. (1970). Silence. Paris : Denoël.

Collectif. (1997). Nomad's land \# 1. Paris : Éditions Kargo.

Cox, C., \& Warner, D. (2004). Audio Culture: Readings in Modern Music. New York : Continuum.

Dagonet, F. (Ed.). (2000). Less is more : stratégies du moins [numéro thématique]. Les Cahiers de Médiologie, 9.

Debray, R. (2000). Ambivalences. Les Cahiers de Médiologie, 9, 19-25.

Deleuze, G., \& Guattari, F. (1998). Mille plateaux. Paris : Editions de Minuit.

DeNora, T. (2000). Music in Everyday Life. Cambridge : Cambridge University Press.

Detry, L. (2002). L'expression musicale du concept de postmodernité par les Beatles: analyse des albums Sgt. Pepper's Lonely Hearts Club Band et The White Album. Mémoire de licence. École de Communication. Louvain-la-Neuve : UCL.

Eno, B. (1996). A Year With Swollen Appendices. London : Faber \& Faber.

Fink, R. (2005). Repeating Ourselves: American Minimal Music as Cultural Practice. Berkeley \& Los Angeles : University of California Press.

Gann, K. (2004). Thankless Attempts at a Definition of Minimalism. Dans C. Cox \& D. Warner (Ed.), Audio Culture: Readings in Modern Music. (pp. 299-303). New York : Continuum.

Lyotard, J.-F. (1996). Musique et postmodernité. Surfaces, VI, 1-11.

McClary, S. (2004). Rap, Minimalism, and Structures of Time in Late TwentiethCentury Culture. Dans C. Cox \& D. Warner (Éd.), Audio Culture: Readings in Modern Music. (pp. 289-298). New York : Continuum.

Mertens, W. (2004). Basic Concepts of Minimal Music. Dans C. Cox \& D. Warner (Éd.), Audio Culture : Readings in Modern Music. (pp. 307-312). New York : Continuum. Mussat, M.-C. (2002). Trajectoires de la musique au XXe siècle. Paris : Klincksieck. Oudin, B. (2000). Architectures minimales. Les Cahiers de Médiologie, 9, 63-71. 
Potter, K. (2007). 1976 and All That: Minimalism and Post-Minimalism, Analysis and Listening Strategies. Dans First International Conference on Music and Minimalism. Bangor.

Ramaut-Chevassus, B. (1998). Musique et postmodernité. Paris : Presses universitaires de France.

Reich, S. (1974). Writings about Music. New York : Universal Edition.

Rosa, H. (2012). Aliénation et accélération: Vers une théorie critique de la modernité tardive. Paris : Éditions La Découverte.

Sacks, O. (2007). Musicophilia. Toronto : Alfred A. Knopf Canada.

Sacks, O. (2011). Awakenings. Toronto : Alfred A. Knopf Canada.

Schwarz, K. R. (1990). Process vs. Intuition in the Recent Works of Steve Reich and John Adams. American Music, 8(3), 245-273.

Stoianova, I. (1977). Musique répétitive. Musique en jeu, 26, 64-74.

\section{Euvres musicales et cinématographiques citées}

Campion, J. (1993). The Piano.

Cage, J. (1952). 4'33".

Eno, B. (1978). Music for Airports.

Glass, P. (1996). Symphony No. 4 « Heroes".

Kubrick, S. (1999). Eyes Wide Shut.

Ligeti, G. (1951-1953). Musica Ricercata.

Reich, S. (1965). It's Gonna Rain.

Reich, S. (1966). Come Out.

Reich, S. (1971). Drumming.

Riley, T. (1964). In C.

Young, L. (1958). Trio for Strings. 